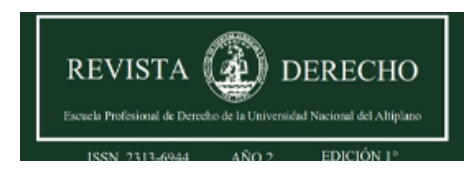

Revista de Derecho

ISSN: 2313-6944

ISSN: 2707-9651

revistaderecho@unap.edu.pe

Universidad Nacional del Altiplano

Perú

\title{
LA EFICACIA DE LAS MEDIDAS DE PROTECCIÓN EN LOS CASOS DE VIOLENCIA INTRAFAMILIAR EN ECUADOR
}

Castillo Martínez, Eduardo Xavier; Ruiz Castillo, Santiago Vinicio

LA EFICACIA DE LAS MEDIDAS DE PROTECCIÓN EN LOS CASOS DE VIOLENCIA INTRAFAMILIAR EN ECUADOR

Revista de Derecho, vol. 6, núm. 2, 2021

Universidad Nacional del Altiplano, Perú

Disponible en: https://www.redalyc.org/articulo.oa?id=671870938009

DOI: https://doi.org/10.47712/rd.2021.v6i2.147

\section{(c) (7)}

Esta obra está bajo una Licencia Creative Commons Atribución 4.0 Internacional. 


\section{LA EFICACIA DE LAS MEDIDAS DE PROTECCIÓN EN LOS CASOS DE VIOLENCIA INTRAFAMILIAR EN ECUADOR}

\section{THE EFFECTIVENESS OF PROTECTION MEASURES IN CASES OF DOMESTIC VIOLENCE IN ECUADOR}

Eduardo Xavier Castillo Martinez

Universidad Técnica Particular de Loja, Ecuador

excastillo@utpl.edu.ec

(iD https://orcid.org/0000-0001-7698-7372

Santiago Vinicio Ruiz Castillo

Universidad Técnica Particular de Loja, Ecuador

svruiz@utpl.edu.ec

(iD) https://orcid.org/0000-0001-9203-6884
DOI: https://doi.org/10.47712/rd.2021.v6i2.147

Redalyc: https://www.redalyc.org/articulo.oa? $\mathrm{id}=671870938009$

\section{Resumen:}

El presente trabajo académico, tiene como objetivo realizar un análisis respecto de la eficacia de las medidas de protección, específicamente en temas de violencia intrafamiliar, las consecuencias que trae consigo un mal uso de las medidas de protección y la falta de un seguimiento oportuno por parte del Estado ecuatoriano. Es importante recalcar que, el Estado como garante de derechos es el encargado de velar por la seguridad y la integridad de la familia ecuatoriana, así como, de resguardar sus derechos y garantías constitucionales; pues, es evidente que en nuestro medio la violencia familiar es un tema que crece a pasos agigantados día a día, y es por ello precisamente que se hace necesaria la oportuna actuación del Estado ecuatoriano.

Palabras claVe: Violencia intrafamiliar, derechos constitucionales, medidas de protección, integridad, mujer, familia.

\section{Abstract:}

The objective of this academic work is to carry out an analysis regarding the effectiveness of protection measures, specifically in matters of domestic violence, the consequences of a misuse of protection measures and the lack of timely monitoring by of the Ecuadorian State. It is important to emphasize that, as a guarantee of rights, the State is in charge of ensuring the safety and integrity of the Ecuadorian family, as well as safeguarding their rights and constitutional guarantees; Well, it is evident that in our environment family violence is an issue that grows by leaps and bounds day by day, and that is precisely why the timely action of the Ecuadorian State is necessary.

KeYwORDS: Domestic violence, women, constitutional rights, protection measures, integrity, family.

\section{INTRODUCCIÓN}

La violencia intrafamiliar es un problema que actualmente forma parte de la sociedad en general, especialmente en épocas de pandemia, afectando en la mayoría de los casos a las mujeres, la Organización Mundial de la Salud (Organización Mundial de la Salud, 2000), menciona que una de cada cinco mujeres en el mundo fue objeto de violencia en alguna etapa de su vida.

No es de sorprendernos que, la mujer a lo largo de la historia se ha mantenido en una lucha constante para proteger y salvaguardar sus derechos ante sujetos opresores; pues, la mujer no sólo ha tenido que sufrir la vulneración de sus derechos en el ámbito familiar, sino también, económico, laboral -moobbing-. La violencia 
intrafamiliar, es un tema, que nace en el núcleo familiar, y en muchos casos se ha naturalizado, y hoy por hoy se lo ve como algo normal.

Al hablar de violencia intrafamiliar se debe tener claro que, no solamente tiene que ver con golpes o agresiones físicas, o sexual, sino, qué también pueden ser golpes emocionales, que afectan la psicopatología individual del individuo, por ejemplo, se ha reportado que los niños que son testigos o víctimas directas de violencia presentan altas probabilidades de padecer depresión y otros tipos de sintomatología emocional (Hundek, 2010. Pp. 70).

Por ello, y ante la falta de leyes encaminadas a la protección de los derechos de la mujer, se implementan en nuestro país normas que tienen como objeto la protección de la mujer, así como, de todas las personas que forman parte del núcleo familiar. Es así que, en la Constitución del 2008 ya se observan algunos articulados enfocados en una vida libre de violencia, protegiendo su seguridad, la integridad física, psicológica y sexual de las personas.

Con estos antecedentes, y con la finalidad de ahondar en el tema propuesto, se tratarán de forma resumida temas como: la violencia intrafamiliar, conceptos y antecedentes, marco legal; medidas de protección, características, el proceso Judicial para el otorgamiento de las Medidas de Protección; eficacia de las medidas de protección; y conclusiones.

\section{LA VIOLENCIA INTRAFAMILIAR}

Es necesario saber de forma clara qué es la violencia intrafamiliar, y de esta forma entender y poder determinar desde nuestra propia perspectiva los aspectos que engloban el tema planteado; así mismo, es importante mencionar algunos antecedentes que tienen que ver con la creación de normas para frenar con la violencia intrafamiliar; y, finalmente el ámbito legal y las diferentes leyes que la engloban.

\subsection{CONCEPTOS}

En palabras de los especialistas Madeline Espinosa, Mercedes Alazales, Bernardo Madrazo, Ada García y María Presno, la violencia intrafamiliar se entiende como la totalidad de situaciones violentas que tienen cabida dentro del hogar, se asume que cualquier miembro puede ser dañado y cualquiera puede dañar, sin embargo, los grupos más afectados y hacia dónde ocurre el daño en mayor frecuencia, recae en las mujeres y los niños (2011).

En este mismo sentido, respecto a la violencia intrafamiliar es toda acción u omisión que se ha cometido en el seno familiar por uno de sus miembros, y menoscaba la vida, la integridad física, psicológica o incluso la libertad de otro miembro de la familia, lo cual causa un daño al desarrollo de la personalidad (Faraoni, 2008. Pp. 393).

Los autores señalados anteriormente, concuerdan al indicar que la violencia intrafamiliar es aquella que surge dentro del núcleo familiar, causada por uno o varios de sus miembros hacia otro miembro de la misma familia, lo cual, radica en la vulneración de sus derechos.

Ahora bien, en este punto es importante indicar lo señalado en nuestro Código Orgánico Integral Penal, respecto a la violencia intrafamiliar:

Se considera violencia a toda acción que consista en maltrato físico, psicológico o sexual ejecutado por un miembro de la familia en contra de la mujer o demás integrantes del núcleo familiar. Se consideran miembros del núcleo familiar a la o al cónyuge, a la pareja en unión de hecho o unión libre, conviviente, ascendientes, descendientes, hermanas, hermanos, parientes hasta el segundo grado de afinidad y personas con las que se determine que el procesado o procesada mantenga o haya mantenido vínculos familiares, íntimos, afectivos, conyugales, de convivencia, noviazgo o de cohabitación (2014. Art. 155). 


\subsection{ANTECEDENTES}

En el año de 1994 se inaugura en nuestro país la primera Comisaría de la Mujer (Acuerdo Ministerial \# 3548 del Ministerio de Gobierno y Policía), en la ciudad de Quito, estas comisarías surgen con el objeto de atender denuncias de mujeres maltratadas y brindarles apoyo legal, psicológico y social de manera eficaz, evitando los trámites que los juzgados civiles debían realizar y con ello una pérdida de tiempo.

Las funciones de estas primeras Comisarías eran limitadas, pues no existía un marco legal que avale la administración de justicia en temas de violencia intrafamiliar, pues no se contaba con una Ley que respalde su gestión.

Es así que, en el año de 1995 se crea la Ley contra la Violencia de la Mujer y la Familia, conocida como Ley 103 (Ley 103 Contra la violencia a la mujer y la familia, 1995); y por primera vez en nuestra legislación se reconoce que la violencia intrafamiliar es un problema social y se asume la responsabilidad de la prevención y sanción a través de la ley (Tamayo, 1998. PP. 29).

Las Comisarías de la Mujer en conjunto con la Ley 103 son los primeros avances en temas de violencia intrafamiliar que se dan en nuestro país, como mecanismo de defensa de derechos y garantías de la mujer, y con la finalidad de lograr una vida sin violencia. Es precisamente aquí dónde se instauran por primera vez las conocidas medidas de amparo, siendo la más común la boleta de auxilio.

A continuación, en el 2013, se crean las primeras Unidades Judiciales de Violencia contra la Mujer y la Familia, y el 10 de agosto del 2014 el Código Orgánico Integral Penal COIP -norma aplicable para casos de contravenciones y delitos de violencia- sustituye a la Ley 103.

El Art. 558 del COIP contiene 12 medidas de protección aplicables en casos de violencia intrafamiliar, las mismas que se resumen de la siguiente manera: alejamiento del agresor con respecto a la víctima, extensión de boleta de auxilio, orden de salida del domicilio de la víctima por parte del presunto agresor, reintegro de la víctima a su domicilio, privación de la custodia temporal de los menores a la persona procesada, suspensión de tenencia de armas al agresor, tratamiento respectivo de acuerdo al caso, suspensión temporal de la actividad contaminante cuando hay riesgo a las personas, orden de desalojo, fijación de una pensión de subsistencia a las personas afectadas por la agresión (Código Orgánico Integral Penal, 2014. Art. 558).

El Registro Oficial Nro. 175 de fecha 05 de febrero del 2018 mediante el cual se crea la Ley Orgánica Integral para prevenir y erradicar la violencia contra las Mujeres, a través de la Cuarta Disposición Reformatoria agrega el Art 558.1 al Código Orgánico Integral Penal, añadiendo 3 medidas de protección en casos de violencia contra las mujeres, las cuales se resumen en: Acompañamiento de los miembros de la Policía Nacional con la finalidad de que las víctimas tome sus pertenencias; orden de que la persona agresora devuelva de forma inmediata objetos de uso personal y documentación personal de la víctima; y, las personas víctimas de violencia de género podrán solicitar, antes, durante y después del proceso penal, el ser incluidas en el programa de protección y asistencia de víctimas a testigos, siempre que fuere el caso y las condicione lo permitieran.

Del mismo modo, el Pleno del Consejo de la Judicatura a través de la Resolución Nro. 154-2014, resolvió expedir "Protocolos para la gestión judicial, actuación y valoración pericial en casos de violencia contra la Mujer o miembros del núcleo familiar", para la atención de víctimas de violencia intrafamiliar, de tal forma se creó la Oficina de Primera Acogida, la cual cuenta con personal capacitado para atender a las personas que llegan a las oficinas en búsqueda de ayuda.

Este personal es el encargado de dotar de la información necesaria y pertinente a la víctima, de indicarle sus derechos, en caso de ser necesario la guía para ser evaluada por el equipo técnico (médico legal, psicólogo, trabajador social) pertinente de acuerdo al caso, además, de informar cual es el procedimiento legal luego de que la denuncia ha sido ingresada.

En este sentido, la denuncia presentada por la víctima se puede ingresar sin el acompañamiento de un abogado, por lo que el papel que desempeña la Oficina de Primera Acogida cumple un rol imprescindible 
de cara a la víctima, mientras que el Estado le brindará un abogado de oficio, siendo éste un profesional especializado en la materia de violencia intrafamiliar para la defensa de los derechos de su defendida o defendido.

\subsection{MARCO LEGAL}

Dentro del marco legal en el cual se introducen las medidas de protección tenemos por ejemplo la Constitución de la República del Ecuador del 2008, específicamente los artículos Art. 11.2 garantiza el derecho a no ser discriminadas por ninguna razón y obliga al Estado a realizar acciones afirmativas para promover una igualdad real a favor de titulares de derechos que se hallen en situación de desigualdad, como es el caso de las mujeres, niños, niñas, adolescentes, personas de la tercera edad y con discapacidad; Art. 66 garantiza los derechos a la integridad física, psíquica, moral y sexual, así como, una vida libre de violencia en el ámbito público como privado; Art. 75 que garantiza el derecho a acceder gratuitamente a la justicia, y a la tutela judicial efectiva, imparcial y expedita; Art. 78 garantiza la adopción de mecanismos para la reparación integral de derechos que han sido violentados.

A nivel internacional tenemos la Convención sobre la eliminación de todas las formas de discriminación contra la mujer (CEDAW, 1981) artículos 1, 6, 11, 12, 13, 14, 15, y 16, estos articulados tratan sobre los derechos y garantías en los casos de violencia doméstica.

Así mismo otra de las normas internacionales de relevancia a nivel continental lo es la Convención BELEM DO PARÁ (1995), Artículo 7 que trata sobre el compromiso de los Estados miembros a condenar la violencia contra la mujer y que estos adopten medidas para erradicarla.

Desde el continente europeo tenemos el Estatuto de la Victima de la Unión Europea (2012), que establece normas sobre los derechos, apoyo y protección de las víctimas.

La Ley de medidas de protección de la Unión Europea (2004), instaura parámetros para otorgar medidas de protección en los casos de violencia doméstica.

En cuanto a la realidad ecuatoriana, y luego de habernos referido en primer lugar a nuestra constitución, la normativa en relación a la protección contra la violencia de la mujer y la familia, se desarrolla actualmente en el Código Orgánico Integral Penal COIP (2014), norma aplicable en contravenciones y delitos de violencia intrafamiliar: Artículos 441, 155, 156, 157, 158, 159, en los cuales se señala quienes son considerados víctimas, además, se establece la violencia física, psicológica y sexual, así como las contravenciones contra la Mujer o miembros del núcleo familiar.

Más adelante en el articulado 558 y 558.1 se señalan con detalle doce medidas de protección, que el juez de acuerdo a su criterio puede otorgar a las víctimas de violencia intrafamiliar, las cuales, fueron ampliadas en el Art. 558.1 conforme la reforma realizada al COIP en el año 2018.

Finalmente, la Ley para Prevenir y Erradicar la Violencia contra las Mujeres (2018), tiene el carácter de orgánica, pues, sus disposiciones prevalecerán sobre otras normas, es importante señalar también, que la presente Ley recoge disposiciones de tratados internacionales, y en cuanto a las medidas de protección, se han ampliado dentro de este cuerpo legal.

\section{LAS MEDIDAS DE PROTECCIÓN}

Las medidas de protección son aquellos medios con las que el juez o jueza dispone para proteger a las víctimas de violencia intrafamiliar, las cuales, deben ser dispuestas de forma inmediata, una vez que el juez o jueza tenga conocimiento de la causa, pues, su objetivo principal es proteger los derechos y garantías de las víctimas. 
En este sentido, Marín López refiere que "las medidas de prevención irán encaminadas a detectar y conocer las causas y efectos de las situaciones de riesgos en que se encuentren las víctimas y posibles víctimas de violencia de género, así como a evitar que les causen daños efectivos" (Marín, 2014. Pp. 466).

En palabras de Alení Díaz Pomé, sobre las medidas de protección y la intervención del Estado al respecto, nos dice:

"Las medidas de protección son aquellas actitudes y decisiones que toma en cuenta el Estado a través de sus diversas instituciones públicas, a fin de hacer efectivo el cuidado y protección de la víctima de la agresión; son mecanismos que buscan brindar apoyo y protección a las víctimas de las agresiones e impedir la continuación de estas".

Es importante resaltar lo referido por Alení Díaz Pomé, quien sobre la intervención del Estado en relación a las medidas de protección “...a fin de hacer efectivo el cuidado y protección de la víctima...”, en la realidad procesal ecuatoriana, el juez especializado en la materia, realiza un seguimiento de las medidas de protección otorgadas a la víctimas, con la finalidad de que las mismas sean cumplidas tanto por la víctima como por el presunto agresor, y esto, se puede lograr a través del apoyo e intervención de instituciones como la Policía Nacional, por medio del Departamento de Violencia Intrafamiliar - DEVIF, el MIES y el Ministerio de Salud Pública a través de sus diferentes departamentos tales como, psicología y trabajo social.

Se debe recordar que las medidas de protección, si bien, van encaminadas a la protección de la víctima, esto, no significa que la víctima puede hacer un uso abusivo o indebido de la mismas, pues, se han dado casos en los cuales, las presuntas víctimas abusan de una de las medidas de protección más comunes en nuestro medio como lo es la boleta de auxilio (COIP, Art. 558.4), por ejemplo, la utilizan para sacar del domicilio al presunto agresor, siendo que el juez no dictó como medida la salida del presunto agresor, amenazándolo con llamar a la policía, refiriendo la existencia de la boleta de auxilio, sin embargo, la medida específica de salida del hogar no había sido dispuesta conforme el artículo ya señalado, siendo que esta última medida sí se establece claramente en la norma en el COIP, Art. 558, numeral 5.

Otro ejemplo lo encontramos cuando existe la presencia de hijos menores de edad como parte de los miembros del núcleo familiar, siendo que las víctimas suelen utilizar las medidas de protección para no dejar ver a los menores por el padre o madre que se constituye en el presunto agresor, en cuyos casos la autoridad competente, tampoco ha ordenado como medida de protección la privación de la custodia temporal de los menores (COIP, Art. 558.7), sin embargo, nuevamente la boleta de auxilio sirve como pretexto para amenazar al presunto agresor e impedir que vea a sus hijos, cuando se debe entender que la boleta de auxilio, no restringe los derechos del progenitor, presunto agresor, sobre los hijos, sino que prevé protección a la víctima, a menos que la boleta de auxilio especifique la extensión de la medida tanto a la presunta víctima como sobre los hijos.

Es así mismo común que las víctimas, obtengan boletas de auxilio, en las cuáles incluso se ordena el alejamiento del presunto agresor; sin embargo, la presunta víctima sin haber permitido que el proceso judicial concluya, hace caso omiso a las medidas de protección que tiene a su favor, y regresa con el victimario, en cuyo caso, este acto se constituye en el incumplimiento de las medidas ordenadas por la autoridad competente, por parte de la misma víctima.

E incluso, existen casos en los que las boletas de auxilio son utilizadas por las víctimas para amenazar al presunto agresor con hacer que este sea recluido en la cárcel, siendo la boleta el medio por el cual la víctima al obtenerla, utiliza mal la misma llamando a la policía, y miente sobre las acciones del presunto agresor, siendo que la policía, en observación de la falta de acatamiento de la boleta, y que deviene en el incumplimiento directo de la orden de un Juez, en consecuencia, aprehende al presunto agresor en el acto para ser puesto a disposición de la autoridad judicial competente y sea el caso remitido como un delito flagrante a ser abordado como tal, aumentándose un proceso penal adicional al ya existente. 
Ante lo señalado en líneas anteriores deviene la importancia de realizar un seguimiento adecuado y oportuno de las medidas de protección, pues de esta forma se lograría una verdadera eficacia en el otorgamiento y cumplimiento de las mismas.

\subsection{CARACTERÍSTICAS}

Como antes se ha indicado, las medidas de protección tienen como finalidad velar por la integridad de la persona, sea física, psicológica o sexual, por ende, tienen el carácter de ser preventivas (Proceso Judicial, Consejo de la Judicatura, Pp. 1 -5).

Además, las medidas de protección son de efecto inmediato, más aún, cuando existe alguna vulneración a la mujer víctima de violencia.

De acuerdo a la Ley Orgánica para prevenir y erradicar la Violencia contra las Mujeres, las medidas de protección pueden ser otorgadas por los Tenientes Políticos a nivel Parroquial; y, a nivel cantonal por las Juntas Cantonales de Protección de Derechos.

Entre las medidas de protección señaladas en el Art 558 del COIP, la más solicitada por las víctimas es la boleta de auxilio, pues, es un documento a través del cual la víctima se siente protegida, ya que, al repetirse una agresión, esta puede llamar a la Policía Nacional, y solo con el hecho de mostrar dicho documento (boleta de auxilio) y ante la transgresión de la misma, el agresor o agresora puede ser aprehendido de forma inmediata.

La boleta de auxilio es una de las medidas de protección más solicitadas y otorgadas en los casos de violencia intrafamiliar, en otras legislaciones se la conoce también como orden de protección (Villagrasa, 2007. PP. 510).

El incumplimiento de las medidas de protección, puede ser sancionado con pena privativa de libertad de 1 a 3 años, de acuerdo a lo que establece el Art. 282 del COIP.

El otorgamiento de las medidas de protección y su aplicación queda a criterio del juez o jueza quien conoce la causa, este es un grave suceso para Barragán (2017) quien denota la relevancia y preocupación al respecto, ya que en su criterio, para que el Juez pueda emitir medidas de protección de forma oportuna y ágil es necesaria una apropiada "preparación, conocimiento académico y empatía humana, transversalizada con experiencia y Derechos Humanos” (PP. 27).

\subsection{PROCESO JUDICIAL PARA EL OTORGAMIENTO DE LAS MEDIDAS DE PROTECCIÓN}

Dependiendo del caso, la denuncia por violencia intrafamiliar puede presentarse en las Unidades Judiciales de Violencia contra la Mujer y la Familia, Unidades Multicompetentes, o si se trata de un delito (Ejemplo: sexual) en la Fiscalía.

Para realizar este tipo de denuncias no se necesita de un abogado, y puede ser presentada de forma verbal o escrita.

La atención a víctimas de violencia intrafamiliar es prioritaria, para ello la oficina de primera acogida deberá orientar a la víctima sobre el proceso judicial, y derivará a la misma en caso de ser necesario a la oficina de atención médica; así la oficina de primera acogida recibirá la entrevista y tomarán la denuncia, para luego continuar con el trámite correspondiente.

En la Unidad Judicial el juez o jueza evaluará el nivel de riesgo en el que se encuentra la víctima al momento de realizar la denuncia, y según el tipo de violencia otorgará las medidas de protección respectivas, mismas que serán emitidas de forma inmediata. 
Si la denuncia de violencia deviene en una contravención, la víctima, debe conocer que más adelante deberá rendir su versión ante el juez o jueza; colaborar con exámenes psicológicos, sociales dependiendo de las disposiciones del juzgador; y, comparecer a la audiencia de juzgamiento acompañado de un abogado defensor.

Este tipo de procesos termina con sentencia del Juez o Jueza, quien, se pronunciará sobre las medidas de protección otorgadas en un inicio, y de ser necesario se ratificará en las mismas, pudiendo incluso extenderlas dependiendo de la gravedad, caso contrario, las dejará sin efecto.

\section{EFICACIA DE LAS MEDIDAS DE PROTECCIÓN}

Para Norberto Bobbio el principio de eficacia se refiere a "determinar si la norma expedida es cumplida a cabalidad por las personas a quienes la ley determina el cumplimiento de una determinada situación”.

En este contexto, debemos recordar lo manifestado por la Constitución de la República del Ecuador, que señala: "La administración pública constituye un servicio a la colectividad que se rige por los principios de eficacia, eficiencia, calidad, jerarquía, desconcentración, descentralización, coordinación, participación, planificación, transparencia y evaluación" (Art. 227), pues, como se ha indicado es el Estado a través de las diferentes instituciones gubernamentales, el encargado de velar por el cumplimiento de las disposiciones de un juez, en el caso que nos ocupa, es quien debe garantizar que las medidas de protección sean cumplidas por las partes, de tal forma que, las mismas cumplan su objetivo, que es, la protección de derechos y garantías constitucionales.

De lo antes manifestado, resulta importante que exista un seguimiento de las medidas de protección, pudiendo de esta forma garantizar su eficacia y evitando así un uso indebido de las mismas.

El abuso de las medidas de protección por parte de los sujetos procesales -víctima o agresor-dentro de un caso de violencia intrafamiliar, pueden devenir en la vulneración de otros derechos, como, por ejemplo, el derecho al libre tránsito, a la propiedad, al trabajo, entre otros.

Se debe entender que la emisión de medidas de protección, no significa para el agresor o agresora la determinación de culpabilidad de la misma, pues las medidas de protección son de carácter preventivas, es decir, lo que se quiere evitar es el cometimiento de nuevos actos de violencia; de ahí que es importante realizar un seguimiento a las medidas de protección emitidas para ambas partes procesales.

\section{CONCLUSIONES:}

- Las medidas de protección en violencia intrafamiliar buscan cumplir con el único fin de evitar nuevos hechos de violencia.

- Si bien las medidas de protección sirven como un medio de seguridad para la víctima, éstas suelen ser mal utilizadas por parte de sus peticionarias, siendo que en muchas ocasiones las medidas son incumplidas por la misma parte interesada.

- La atención que deben recibir las víctimas de violencia debe ser oportuna y eficaz, y con personal especializado.

- La falta de capacitación puede desencadenar en la falta de un seguimiento en los casos de violencia, y en especial atención al cumplimiento de las medidas de protección; pues la falta de seguimiento a su cumplimiento provocaría una serie de violaciones de derechos tanto para la víctima como para el presunto agresor.

- Es necesario que exista un seguimiento por parte de las instituciones del Estado, en los casos de violencia intrafamiliar, este seguimiento, deberá concluir una vez concluya el proceso judicial. 


\section{REFERENCIAS BIBLIOGRÁFICAS}

Barragán, A. (2017). El seguimiento a las medidas de protección otorgadas en casos de violencia intrafamiliar en la unidad judicial segunda de violencia contra la mujer y la familia, en el periodo comprendido entre julio y diciembre del 2016. Universidad Central del Ecuador. Pp. 27.

Bobbio, N. Contribución a la Teoría General del Derecho. Editorial Debate. Madrid, 1990. Pp. 57, 58.

Espinosa Morales, M; Alazales Javiqué, M; y, otros. (2011). Violencia Intrafamiliar, realidad de la Mujer Latinoamericana. Cuba. Revista Cubana de Medicina General Integral. Marzo del 2011.

Faraoni, F. (2008). Derecho de Familia Visión Jurisprudencial. Córdova - Argentina. Editorial, Nuevo Enfoque Jurídico. Pp. 393.

Hundek Pichón, L. (2010). Violencia Doméstica: hombres versus mujeres maltratantes en la ciudad de Barranquilla. Revista Pensamiento Americano, Nro. 4, junio 2010. Pp. 71.

Marín López, P. (2014). Tutela Judicial. Navarra. Editorial Aranzadi S.A. Medidas de Protección. Disponible en la URL: https://trabajadorjudicial.wordpress.com/la-efectividad-de-las-medidas-de-. proteccion-frente-a-laviolencia-familiar . Consultado el 03 de junio de 2021.

Organización Mundial de la Salud: conclusiones del Foro Día Internacional para la Eliminación de la Violencia Contra las Mujeres, 24 de nov. 2000.

Tamayo, C. (1998). Investigación de Impacto proyecto de fortalecimiento de las Comisarías de la Mujer y la Familia. Centro Ecuatoriano de Protección y Acción de la Mujer - CEPAM. Diciembre 1998. Pp. 29.

Villagrasa Alcaide, C. (2014). Protección en el ámbito civil. Navarra. Editorial Aranzadi S.A.

Constitución de la República del Ecuador. Decreto Legislativo 0. Publicada en el Registro Oficial 449 de 20 de octubre de 2008, última reforma, 30 de abril de 2019.

Código Orgánico Integral Penal. Publicado en el Registro Oficial Suplemento 180, lunes 10 de febrero del 2014.

Ley Orgánica para prevenir y erradicar la Violencia contra las Mujeres. Registro Oficial Suplemento 175 de 05 de febrero del 2018.

Convención sobre la eliminación de todas las formas de discriminación contra la mujer,1946.

Estatuto de la Víctima, del 27 de abril del 2015.

Ley 103 (contra la violencia a la mujer y la familia) y Reglamento. Ministerio de Gobierno, de 1997.

Ley de medidas de protección de la Unión Europea, del 28 de diciembre de 2004.

Convención Interamericana para prevenir, sancionar y radicar la Violencia Contra la Mujer “Belem Do Para”, 1996.

Convención para la Eliminación de todas las formas de Discriminación en Contra de la Mujer, CEDAW, 1998.

Conoce tus Derechos. Consejo de la Judicatura. https://www.funcionjudicial.gob.ec/www/pdf/Conoce\%20tus \%20derechos-08-03-2016-2.pdf 